\title{
A Review of Stressors as Inducer for Personality Disorders and Anxiety Disorders
}

\author{
Zhegan Shi ${ }^{1 *}$ \\ ${ }^{1}$ International Campus Zhejiang University, ZJU-UoE Institute, Haining City, China, 314400 \\ *Corresponding author. Email: zhegan.18@intl.zju.edu.cn
}

\begin{abstract}
Personality disorders (PDs) and anxiety disorders (ADs) are common mental disorders. PDs are often more or less related to anxiety and ADs are affected by personality functioning. To clarify the pathology of both ADs and PDs, along with their similarities and differences, the literature review by paired keywords search on NCBI PubMed and subsequent analysis are used. The findings support that abnormal stressors can damage the hypothalamus-pituitary-adrenal (HPA) axis time by time throughout life through the epigenetic pathway, lowing down the ability of the stress system to deal with stressors. Biological basis, like the genetic issue, for PDs, is important but after-born experience also matters a lot. Adjoint stressful long-term memory may largely affect normal personality formation, contributing to some kinds of PDs. It seems that early exposure to these stressors influences PDs more, compared to ADs. More researches are needed to explore the effect on ADs from later life stress. This study partially explained the process of ADs and PDs, linked them by abnormal stressors and emphasized the importance of focusing on the effect of stressful long-term memory formation, which is psychological, and the HPA axis disability, which is biological, on patients having these diseases, reminds psychiatrist to treat disease at the source, to low down the potential risk of getting PDs in patients who are diagnosed with ADs, to prevent arouse of ADs in patients who are diagnosed with PDs and to combine biological treatment with psychological therapies.
\end{abstract}

Keywords: personality disorders, anxiety disorders, HPA axis, long-term memory

\section{INTRODUCTION}

In recent years, many facts have been discovered on the relationship between PDs and ADs. Avoidant personality disorder (AVPD) has a close relationship with a social anxiety disorder (SAD) [1] and ADs are often seen in borderline personality disorder (BPD) [2]. In patients with severe health anxiety (SHA), the rate of having PDs is around $71.4 \%$, referred to Structured Clinical Interview for DSM-IV axis II (SCID-II), PID-5 Whiteley Index 7 and Short Health Anxiety Inventory, indicating a common personality pathology with SHA[3]. Personality functioning (PF), a significant requirement for diagnosing PDs, is associated with ADs[4].

Stressors can induce stress responses and subsequent anxiety by affecting the biological system, including the brain, neuroendocrine system and immune system [5]. Anxiety is normally not pathological but maladaptive anxiety, such as long-lasting anxiety, can cause ADs [6]. Stressors exposed to pregnancy lead to an increased risk of postnatal personality disorder in offspring, though independent of psychiatric disorders [7].

Negative childhood experiences, causing dysfunction of the HPA axis [8], can lead to some kind of PD in adults [9]. Early life stress can also induce ADs in later life through changing the status of the HPA axis. Stressors may affect the HPA axis to bring up the PDs and ADs.

Many HPA axis related issues have also been found to have possible responsibilities for the pathology of both PDs and ADs, such as corticotropin-releasing hormone (CRH), neuropeptide Y (NPY), glucocorticoids (GC), different neurotransmitters of monoaminergic neurotransmitter systems and their receptors $[10,11,12]$.

Genetic research has shown some common candidate genes coding for, such as catechol-O-methyltransferase (COMT), monoamine oxidase A (MAOA) and serotonin-transporter-linked promoter region (5-HTTLPR), are abnormal in both ADs and one sub-cluster of PDs. These genes are 
involved in the neurotransmitter pathways. Epigenetic research has further explained how stressors affect gene expression $[13,14]$.

The Hippocampus, which is an important part of the limbic system for long-term memory formation, has been mentioned by some research to matter, indicating stressful long-term memory may as another kind of possible stressor, instead of environmental factors, to influence the buildup of PDs and ADs.

This study aims to give a possible explanation for the high rate of comorbidity of PDs and ADs about their relationship based on current research findings and common views and give a suggestion for future research and clinical diagnosis and therapies.

\section{BIOLOGICAL AND PSYCHOLOGICAL MECHANISMS OF ADS AND PDS}

\subsection{Stress controllability}

The stress response is a bidirectional process that includes how severe the stressors are that are perceived and cognized by people and how the stress system can deal with them. The same stressor may be processed to be more or less stressful due to some factors, such as the long-term memory from former events, by a person. Stress controllability, which is discovered as how a person can control the outcome of a stressful event. High stress controllability contributes to good performance in subsequent fear extinction trial, though increases "flight" behaviour in irrelevant stress context, while low stress controllability leads to slakeless stress response, representing a prolonged stressor cognition or stressful emotion based on long-term memory about inevitable stress outcome [15].

\subsection{Stress resilience}

The psychological dimension of stress resilience refers to how a person cognizes a perceived stressful event, rationally or sentimentally, negatively or positively, due to his/her personality, which is also affected by personal long-term memory. This kind of cognition influences the severity of an objective stressor, or the income of a stressful event, subjectively. The biological dimension of stress resilience is the ability to pull down the stress response, which is mainly built up by the HPA axis. Cognized stressors trigger the secretion of $\mathrm{CRH}$ in the paraventricular nucleus of the hypothalamus. CRH is the main factor controlling the activity of the HPA axis, which finally induces cortisol secretion to cause metabolic change and stressful symptoms. Many neurotransmitters, related enzymes, transporters and receptors regulate the secretion of CRH. 5-hydroxytryptamine (5-HT) or serotonin is a neurotransmitter that activates $\mathrm{CRH}$ secretion and is synthesized from tryptophan (TRP) by the enzyme TPH.
Damage of TPH leads to disability of coping with uncontrollable stress. The aminobutyric acid (GABA) system, the dopaminergic system, the norepinephrine system, etc., also can modulate stress resilience through regulating CRH secretion. The downstream of the HPA axis is also important as the negative feedback mechanism of cortisol can inhibit excessive CRH secretion by binding to mineralocorticoid receptors (MRs) and glucocorticoid receptors (GRs). NPY is also found to benefit stress resilience but the underlying mechanism still needs to be further explored $[16,17]$.

\subsection{Genetic and epigenetic issues}

Genes, encoding for CRH, ACTH, cortisol and the regulators of $\mathrm{CRH}$, their precursors, related enzymes, their transporters, receptors, decide the original stress processing capacity of the HPA axis. During development, external stressors from the environment or internal stressors from long-term memory modulate the capacity, through epigenetic pathways, such as DNA methylation, and these kinds of changes are long-lasting, even heritable. For example, stressor exposure to pregnant women may change the gene expression of the embryo, though the gene sequences are not changed, the differentiation of the embryo for HPA axis formation is changed, leading to an altered basis of HPA function, and during childhood development, epigenetic pathways continue to play a role. The change of the activity of DNA and the structure of the chromatin can be stable, and the intensity and duration of the stressors may be to blame. That is why an early life trauma or sustained childhood abuse can increase the risk of getting ADs in later life, as the ability of the HPA axis to relieve stress response is weakened, feels of anxiety increase.

\subsection{Personality formation}

As mentioned above, memory plays an important role in personality. In some way, personality formation can get clues from the process of long-term memory formation, including perception, processing of perception and cognition with or without emotion or will. Most perception is sensory or short-term memory but through processing, it can become long-term memory. Perception processing often refers to the use of thinking patterns or models, which may be based on the co-activation of specific brain areas, and so-called long-term memory is then formed. Long-term memory can be implicit or explicit. Thinking patterns or models are implicit long-term memory, people cannot explain clearly how they process what they perceived but they know they should or could deal with the information in that way or even unconscious of that they have dealt with the information in that way. Cognition is explicit long-term memory. Cognition is processed perception, in other words, the conclusion of perception. However, 
not all perception needs to be processed to be cognition, some perception is just memorized as cognition, and, existed cognition is often used to form new cognition during the processing of perception. Emotion and will are related to cognition but still not known clearly.

Personality is a series of thinking patterns or models directing cognition, emotion, will and subsequent behaviour, suggesting that personality formation may be partially based on long-term memory.

The processing of perception is not an independent system, in other words, the thinking patterns or models are affected by perception and cognition with or without emotion or will. What is perceived is and the time course matter. As for some kinds of PDs, excessive stressors are perceived, especially in early life.

\section{THE EXPLANATION FOR A SINGLE DISEASE OF ADS OR PDS}

During childhood development, abnormal stressors can cause stressful long-term memory, as well as stable biological change of the HPA axis. Stressful long-term memory is an important factor affecting personality formation, increasing the risk of getting PDs. Personality is stable, a well-established personality is resistant to later personality change due to stressful events, often with a good HPA axis condition as well. However, when facing stressors that are too intense, long-lasting or both for normal HPA axis to deal with, people without PDs still can get ADs. People with PDs are often more sensitive to stressors and their capacity of the HPA axis to cope with stressors are weak, increasing the risk of getting $\mathrm{ADs}$, consistent with the fact that a high co-occurrence of PDs and ADs. Again, exposure to abnormal stressors is important, people with PDs still have a chance to avoid ADs.

\section{DISCUSSION}

Many pieces of research have been done on negative feedbacks or the mechanisms to drawback stress response, which is very important in the pathology of ADs, but the arousal of stress is also important, which is less researched. Recall of stressful long-term memory may aggravate the cognition of the stressors that are facing and thus cause a higher level of stress arousal. Long-term memory may affect the HPA axis in this way.

Personality formation is a complex process. Besides long-term memory that got from interactions with the natural or social environment, genetic issues are the major one that is always mentioned, which can influence the intelligence, an important factor to form cognition, of a person, but intelligence still relates to long-term memory.

Many opinions have yet to be confirmed. The explanation for personality formation is lack of related research findings but is still reasonable as is based on common views on long-term memory that from brain science research. PDs and ADs share so many correlations may be because they have the same inducer that is the stressor. Abnormal stressor physically causes HPA axis capacity damage, psychologically causes stressful long-term memory, but the time course matters, exposure in the early stage of life causes the both, while exposure after personality formation seems to only cause the physical result. PDs seem to have close relations with stressful long-term memory and the HPA axis abnormality simultaneously.

Psychological researches are needed to explore whether the hippocampus does be activated during abnormal stressor exposure in early life and which brain areas are co-activated to store the stressful long-term memory, whether these brain areas are re-activated in later stressful events. More biological researches are needed to figure out the possibility and method of normal function regeneration of the HPA axis, but not creating drugs that just relieve the symptoms, though easing the pain of patients is also meaningful.

Patients with ADs are more likely to ask for help from doctors than those with PDs due to the severity of symptoms. It is important to diagnose the existence of PDs in ADs patients, but the time of the initiation of PDs need to be considered as mentioned above and according to the diagnostic criteria of ICD-10 and DSM-IV. It is more important to measure the HPA axis function after a patient has only been diagnosed with PDs to give early diagnosis and treatment for ADs.

\section{CONCLUSION}

Early-life exposure to stressors can modulate the HPA axis epigenetically based on a genetic foundation and form implicit and explicit long-term memory. This may explain why stressful experience in early life is so important for the formation of the stress system. Abnormal stressors, on a time scale, like acute or chronic stress events before adulthood, can damage normal functions of the HPA axis long, through upregulating stress arousal and downregulating stress repression. It is admitted that personality is relatively stable after formation so early life experience may affect a lot. The memory to these abnormal events, mainly implicit long-term memory, may largely affect the formation of normal personality, leading to unsuited behaviour based on formed strategies, which are based on former life experience, here like stress strategy that to avoid or cope with inevitable stress events, and can also contribute to an out-of-control stress response.

Later life exposure to stressors may also matter especially for ADs, but there is little research as many epidemiology investigations on ADs have shown that the young is the most susceptible subject. A dynamic 
balance probably exists between stressor exposure and stress inhibition capability during the whole lifetime. Early exposure to abnormal stressors decreases the upper limit of the capability, so it is easier to get ADs in later life but later exposure can also constantly harm the stress system to increase the risk of getting ADs after, for some research has shown the prevalence in older people is the highest. Thus, it seems that it can be a good way to divide the stress exposure in the course of ADs by "early" or "later" only if there is a significant difference in biological change like the HPA axis that is affected by a first-time exposure and later ones. The common stressors that are faced within different life stages may need to be more considered, which is an important factor to influence the prevalence.

This paper figures out that why ADs and PDs are so correlated and reminds psychiatrist to treat disease at the source, to low down the potential risk of getting PDs in patients who are diagnosed with ADs, to prevent arouse of ADs in patients who are diagnosed with PDs and to combine biological treatment with psychological therapies. Besides, future research on later life stress is recommended.

\section{AUTHORS' CONTRIBUTIONS} Shi.

This paper is independently completed by Zhegan

\section{ACKNOWLEDGMENTS}

This paper is carried out with the inspiration that is based on the online course named Language and Memory in Developmental Psychology, which is organized by Prof. Szucs, Reader in Cognitive Neuroscience and Psychology, University of Cambridge, UK and Master Zhou, University of Chinese Academy of Science.

\section{REFERENCES}

[1] Weinbrecht, A., Schulze, L., Boettcher, J. et al. Avoidant Personality Disorder: a Current Review. Curr Psychiatry Rep 18, 29 (2016). https://doi.org/10.1007/s11920-016-0665-6.

[2] Quenneville AF, Kalogeropoulou E, Küng AL, Hasler R, Nicastro R, Prada P, Perroud N. Childhood maltreatment, anxiety disorders and outcome in borderline personality disorder. Psychiatry Res. 2020 Feb, 284:112688.

[3] Skjernov M, Bach B, Fink P, Fallon B, Soegaard U, Simonsen E. DSM-5 Personality Disorders and Traits in Patients With Severe Health Anxiety. J Nerv Ment Dis. 2020 Feb, 208(2):108-117.

[4] Gruber, Maria; Doering, Stephan; Blüml, Victor Personality functioning in anxiety disorders,
Current Opinion in Psychiatry: January 2020 Volume 33 - Issue 1 - p 62-69.

[5] A. Ray, K. Gulati, N. Rai, Chapter One - Stress, Anxiety, and Immunomodulation: A Pharmacological Analysis, Editor(s): Gerald Litwack, Vitamins and Hormones, Academic Press,Volume 103, 2017, Pages 1-25, ISSN 0083-6729, ISBN 9780128119143.

[6] Beesdo K, Knappe S, Pine DS. Anxiety and anxiety disorders in children and adolescents: developmental issues and implications for DSM-V. Psychiatr Clin North Am. 2009, 32(3):483-524.

[7] Brannigan, R., Tanskanen, A., Huttunen, M., Cannon, M., Leacy, F., \& Clarke, M. The role of prenatal stress as a pathway to personality disorder: Longitudinal birth cohort study. The British Journal of Psychiatry, 216, 2020(2), 85-89.

[8] Dempster KS, O'Leary DD, MacNeil AJ, Hodges GJ, Wade TJ. Linking the hemodynamic consequences of adverse childhood experiences to an altered HPA axis and acute stress response. Brain Behav Immun. 2021 Mar, 93:254-263.

[9] Mainali P, Rai T, Rutkofsky IH. From Child Abuse to Developing Borderline Personality Disorder Into Adulthood: Exploring the Neuromorphological and Epigenetic Pathway. Cureus. 2020 Jul 30, 12(7):e9474.

[10] Claes, S. Corticotropin-releasing hormone (CRH) in psychiatry: from stress to psychopathology. Annals of Medicine, 2004, 36(1), 50-61.

[11] Schmeltzer SN, Herman JP, Sah R. Neuropeptide Y (NPY) and posttraumatic stress disorder (PTSD): A translational update. Exp Neurol. 2016 Oct, 284(Pt B):196-210.

[12] Tsigos C, Chrousos GP. Hypothalamic-pituitary-adrenal axis, neuroendocrine factors and stress. J Psychosom Res. 2002 Oct, 53(4):865-71.

[13] Meier, S.M., Deckert, J. Genetics of Anxiety Disorders. Curr Psychiatry Rep 21, 16, 2019.

[14] Gescher DM, Kahl KG, Hillemacher T, Frieling H, Kuhn J, Frodl T. Epigenetics in Personality Disorders: Today's Insights. Front Psychiatry. 2018, 9:579.

[15] Hartley CA, Gorun A, Reddan MC, Ramirez F, Phelps EA. Stressor controllability modulates fear extinction in humans. Neurobiol Learn Mem. 2014, 113:149-156. 
[16] Maul, S, Giegling, I, Fabbri, C, Corponi, F, Serretti, A, Rujescu, D. Genetics of resilience: Implications from genome-wide association studies and candidate genes of the stress response system in posttraumatic stress disorder and depression. Am J Med Genet Part B. 2020, 183: 77- 94.

[17] Faye C, Mcgowan JC, Denny CA, David DJ. Neurobiological Mechanisms of Stress Resilience and Implications for the Aged Population. Curr Neuropharmacol. 2018, 16(3):234-270. 